\title{
KMC_Lattice v2.0: An Object-Oriented C++ Library for Custom Kinetic Monte Carlo Simulations
}

\author{
Michael C. Heiber ${ }^{1}$ \\ 1 Center for Hierarchical Materials Design (CHiMaD), Northwestern University, Evanston, Illinois \\ 60208, USA
}

DOI: $10.21105 /$ joss. 01168

\section{Software}

- Review ¿

- Repository ${ }^{\boldsymbol{A}}$

- Archive ¿

Submitted: 07 January 2019

Published: 29 January 2019

\section{License}

Authors of papers retain copyright and release the work under a Creative Commons Attribution 4.0 International License (CC-BY).

\section{Summary}

Kinetic Monte Carlo (KMC) simulations are a powerful method for investigating the dynamics of non-equilibrium systems (Voter, 2007) and have been used to help solve problems in a wide variety of scientific domains, including atomic simulations of epitaxial growth (Martin-Bragado, Borges, Balbuena, \& Jaraiz, 2018), vacancy diffusion and grain growth in solids (S. Plimpton et al., 2009), opto-electronic mechanisms in disordered organic electronic devices (Heiber, Wagenpfahl, \& Deibel, 2019), chemical and ionic diffusion and reactions for heterogeneous catalysis (Stamatakis, 2015) and electrochemical systems (Turner, Zhang, Gelb, \& Dunlap, 2015), complex chemical reaction networks (Gillespie, 2007), and predator-prey population dynamics (Dobramysl, Mobilia, Pliemling, \& Täuber, 2018). To tackle this diverse range of problems there are numerous open-source software tools with different levels of quality, flexibility, documentation, testing, and support. Of these, SPPARKS (S. Plimpton et al., 2009, Steve Plimpton, Thompson, \& Slepoy (2009)), kmos (Hoffmann, Matera, \& Reuter, 2014,Hoffmann (2013)), and KMCLib (Leetmaa \& Skorodumova, 2014,Leetmaa (2014)) are several other examples of high-quality general KMC framework software tools for particle simulations. There are also numerous closed-source tools that have been developed by various research groups and companies around the world. However, the KMC_Lattice library is uniquely designed to be a lightweight and flexible object-oriented $\mathrm{C}++$ library that allows developers to more easily create custom KMC simulation software packages for use with high performance computing resources. With detailed API documentation using Doxygen, rigorous testing using googletest, and continuous integration testing using TravisCI, KMC_Lattice is built to be a reliable and scalable package that can be used by a wide variety of other open-source KMC software tools in the future. Currently, I am using the KMC_Lattice library as the foundation for a new KMC software package called Excimontec, which is designed to simulate disordered organic electronic devices (Heiber, 2018).

The KMC_Lattice library contains a number of base (parent) classes that must be extended to create derived (child) classes to implement a user's simulation model of choice. Users must define the object entities in the simulation model and all of the events (transitions) possible for each object type. For each event, users must define the conditions under which each event occurs and can also define custom rate constant functions. Custom rate constant calculation functions can allow complex interactions between objects or between the objects and the simulation environment (lattice sites). KMC_Lattice v2.0 allows users to create simulation models on a cubic lattice and combines the rejectionfree Bortz, Kalos, and Lebowitz (BKL) algorithm (Bortz, Kalos, \& Lebowitz, 1975) and several variations of Gillespie's first-reaction method (Gillespie, 1976) to efficiently propagate the simulation. It is also designed to use MPI functionality to efficiently parallelize 
calculations for gathering statistics of the behavior of the stochastic simulation and contains a number of utility functions for gathering and analyzing the data generated by the simulation. The source code for all releases is archived with Zenodo.

\section{Acknowledgments}

This work was developed under the financial assistance award 70NANB14H012 from U.S. Department of Commerce, National Institute of Standards and Technology as part of the Center for Hierarchical Materials Design (CHiMaD). Thank you to Dr. Dean M. DeLongchamp for providing access to NIST's Raritan computing cluster, which was helpful with software development and testing.

\section{References}

Bortz, A. B., Kalos, M. H., \& Lebowitz, J. L. (1975). A new algorithm for Monte Carlo simulation of ising spin systems. J. Comp. Phys., 17, 10-18. doi:10.1016/0021-9991(75) 90060-1

Dobramysl, U., Mobilia, M., Pliemling, M., \& Täuber, U. C. (2018). Stochastic population dynamics in spatially extended predator-prey systems. J. Phys. A: Math. Theor., 51, 063001. doi:10.1088/1751-8121/aa95c7

Gillespie, D. T. (1976). A general method for numerically simulating the stochastic time evolution of coupled chemical reactions. J. Comp. Phys., 22, 403-434. doi:10.1016/ 0021-9991(76)90041-3

Gillespie, D. T. (2007). Stochastic simulation of chemical kinetics. Annu. Rev. Phys. Chem., 58, 35-55. doi:10.1146/annurev.physchem.58.032806.104637

Heiber, M. C. (2018). Excimontec. https://github.com/MikeHeiber/Excimontec.

Heiber, M. C., Wagenpfahl, A., \& Deibel, C. (2019). Advances in modeling the physics of disordered organic electronic devices. In O. Ostroverkhova (Ed.), Handbook of organic materials for electronic and photonic devices, Woodhead publishing series in electronic and optical materials (2nd ed., pp. 309-347). Woodhead Publishing. doi:10.1016/ B978-0-08-102284-9.00010-3

Hoffmann, M. K. (2013). kmos. https://github.com/mhoffman/kmos.

Hoffmann, M. K., Matera, S., \& Reuter, K. (2014). kmos: A lattice kinetic Monte Carlo framework. Comput. Phys. Commun., 185, 2138-2150. doi:10.1016/j.cpc.2014.04.003

Leetmaa, M. (2014). KMCLib. https://github.com/leetmaa/KMCLib.

Leetmaa, M., \& Skorodumova, N. V. (2014). KMCLib: A general framework for lattice kinetic Monte Carlo (kmc) simulations. Comput. Phys. Commun., 185, 2340-2349. doi:10.1016/j.cpc.2014.04.017

Martin-Bragado, I., Borges, R., Balbuena, J. P., \& Jaraiz, M. (2018). Kinetic Monte Carlo simulation for semiconductor processing: A review. Prog. Mater. Sci., 92, 1-32. doi:10.1016/j.pmatsci.2017.09.003

Plimpton, S., Battaile, C., Chandross, M., Holm, L., Thompson, A., Tikare, V., Wagner, G., et al. (2009). Crossing the mesoscale no-man's land via parallel kinetic Monte Carlo (No. SAND2009-6226). Sandia report. Sandia National Laboratories.

Plimpton, S., Thompson, A., \& Slepoy, A. (2009). SSPARKS. https://spparks.sandia.gov. 
Stamatakis, M. (2015). Kinetic modelling of heterogeneous catalytic systems. J. Phys.: Condens. Matter, 27, 013001. doi:10.1088/0953-8984/27/1/013001

Turner, C. H., Zhang, Z., Gelb, L. D., \& Dunlap, B. I. (2015). Kinetic Monte Carlo simulation of electrochemical systems. In A. L. Parrill \& K. B. Lipkowitz (Eds.), Reviews in computational chemistry (1st ed., Vol. 28, pp. 175-204).

Voter, A. F. (2007). Introduction to the kinetic Monte Carlo method. In K. E. Sickafus, E. A. Kotomin, \& B. P. Uberuaga (Eds.), Radiation effects in solids, NATO science series (Vol. 235, pp. 1-23). Dordrecht: Springer. doi:10.1007/978-1-4020-5295-8_1 